Research Article

\title{
Application of Data Envelopment and Internet of Things Technology for Asset Value Evaluation
}

\author{
Lunsheng Wang, ${ }^{1}$ Mingwu Gao, ${ }^{2}$ and Zhenlu Liang ${ }^{3}{ }^{3}$ \\ ${ }^{1}$ Information Center, Shandong Institute of Commerce and Technology, Jinan 250103, Shandong, China \\ ${ }^{2}$ Publicity Department, Information Center, Shandong University of Arts, Jinan 250014, Shandong, China \\ ${ }^{3}$ Network Information Center, Qilu University of Technology, Jinan 250353, Shandong, China
}

Correspondence should be addressed to Zhenlu Liang; lzl@qlu.edu.cn

Received 1 April 2021; Revised 19 April 2021; Accepted 26 April 2021; Published 4 May 2021

Academic Editor: Mian Ahmad Jan

Copyright (c) 2021 Lunsheng Wang et al. This is an open access article distributed under the Creative Commons Attribution License, which permits unrestricted use, distribution, and reproduction in any medium, provided the original work is properly cited.

\begin{abstract}
Assets are an essential part of an enterprise's production and an indispensable economic entity for production and operation. The valuation of assets is one such area that has received significant attention in recent years. However, there exist numerous issues from various perspectives. These issues require us to study and explore them further. This article discusses the role of Internet of Things (IoT) and data envelopment methods to improve and develop asset evaluation. Based on the concept of asset evaluation, this paper designs an asset evaluation framework using the IoT. This framework can provide an all-weather and no-delay preparation basis for the asset appraisal work. Moreover, this paper uses data envelopment and particle swarm optimization (PSO) algorithm to clean and reclassify asset data. The objective of this algorithm is to solve the nonlinear function optimization problems associated with asset value evaluation. Finally, we verify the effectiveness and practicability of the model by comparing experimental simulation and manual evaluation.
\end{abstract}

\section{Introduction}

In the context of the rapid development of contemporary science and technology in microeconomic activities worldwide, intangible assets show much more vitality than tangible assets [1]. The amount of intangible assets generally reflects the scientific and technological capabilities and intellectual and cultural capabilities possessed by these assets' owners. Intangible assets are an essential part of an enterprise and indispensable in its production and operation $[2,3]$. The country's long-term asset management practices under the conditions of a market economy provide a basis for evaluating intangible assets. The existing economic knowledge and mathematical methods provide a means to establish an evaluation system that can offer intangible assets' value $[4,5]$. With the continuous deepening of reform and opening up and the rapid development of the socialist market economy, intangible assets and their assessment issues have attracted significant attention from relevant state departments. Especially, in the recent period, articles calling for attention and strengthening intangible assets' assessment are not uncommon [6-8]. Through the development of intangible assets' evaluation, the role, status, and value of intangible assets will be recognized and valued by the society. However, this work still has many problems in many aspects [9].

With the advent of globalization and the era of knowledge economy, the profoundly changing domestic and international environment on the one hand provides extensive development space for the asset appraisal industry, but also brings a lot of new problems to the healthy development of asset appraisal industry. The evaluation business has begun to transform from traditional business to emerging business [10]. The types and scope of evaluation have gradually expanded, and the market's requirements for evaluation and quality have become higher and higher $[11,12]$. However, many asset appraisal agencies still adopt the old business philosophy, blindly pursue economic 
benefits, and neglect the control of appraisal risks [13]. Frederick M. Babcock pointed out that it is necessary to determine which evaluation method to choose based on its purpose and the specific asset status [14]. It is necessary to define the purpose of the evaluation before starting the evaluation work. Jaffe and Lush pointed out that a correct understanding of the meaning of value in economic theory can help improve the quality of evaluation results [15]. With the further development of measurement technology and measurement tools, evaluators' evaluation results will be more accurate [16]. The cornerstone of asset appraisal research is the theoretical basis of appraisal. The research on appraisal theoretical basis mainly discusses the problem of value concept and the relationship between appraisal theory and economic theory [17].

The significance of asset evaluation is not to know how much a company's asset brand is worth, but to help companies find the advantages and weaknesses of brand equity, to achieve the benefits of further enhancing brand equity. With the continuous deepening of reform and opening up and the rapid development of the socialist market economy, intangible assets and their assessment issues have attracted significant attention from relevant state departments [18]. The studies mentioned above have many problems and challenges that need to be addressed. These issues require us to study and explore them in practice. This article discusses the use of Internet of Things (IoT) technology, data envelopment methods, and particle swarm optimization (PSO) algorithm to further improve and develop asset evaluation methods to carry out related work on asset evaluation [19]. Data envelopment and PSO algorithm ensure the cleaning and reclassification of asset data. On the other hand, IoT provides the adaptation of the latest technological trends used for asset evaluation within the manufacturing industry.

The rest of this paper is organized as follows. In Section 2, we will give an overview of related technologies, i.e., asset evaluation and Internet of Things. In Section 3, we will introduce the asset evaluation data analysis based on data envelopment and particle swarm. In Section 4, we will perform the performance and simulation tests of the model built in Section 3. Finally, we summarize and discuss the methods of this paper and give future research directions in Section 5 .

\section{Related Work}

In this section, first we provide a brief overview and the latest technological trends in IoT followed by the existing studies for asset evaluation.

2.1. Internet of Things Technology. The productive social forces are constantly developing, and the subsequent demands for various intelligent applications are also being affected by this development. In an environment where multiple technologies collide and blend, the IoT with the goal of "intelligence" has begun to emerge [20]. The IoT"s communication range can cover the world, and data can be exchanged between real objects, and all data sent by real objects can be reflected in real time through legal means [21].
IoT's perception layer is like the nerve endings of the human body's sensory organs, which can connect data in the information space with those in the physical space [22]. The perception layer can obtain the environmental information for various applications, e.g., the manufacturing industry. The information obtained through the built-in special equipment processing responds according to the agreed procedure and transmits the reaction result to the transmission layer through a special channel [23]. The collection devices in the perception layer are called perception devices or perception nodes. Standard devices such as RFID, twodimensional codes, sensor nodes, and smart terminals can be embedded into mobile communication terminals [24]. From the above description of IoT, in a narrow sense, the automatic identification function and intelligent operation of the IoT are smart networks that are connected based on objects and people. The IoT uses numbers and networks to represent all things, achieves the transmission between people and the transmission in objects, and finally integrates the data transmitted between them.

2.2. Asset Valuation. Asset appraisal method is the general term for a series of technical means implemented by appraisers in the asset appraisal business for the purpose of assessing and estimating the assets' value. At present, the three basic evaluation methods commonly used in the world are the cost method, the market method, and the income method [25]. They apply the technical methods in financial management, accounting, engineering technology, statistics, and other disciplines in accordance with the inherent requirements of asset evaluation to the practice of asset evaluation. They are the three major components of the asset evaluation method system [26]. These three basic methods are actually to judge the value of assets from different time points. Based on the value at different time points, the current value of the assessed object is obtained after analysis and calculation. The schematic diagram of asset evaluation methods and characteristics is shown in Figure 1.

From the perspective of asset appraisal, value and price belong to two different conceptual categories. Value belongs to the category of exchange value [27]. It reflects the monetary relationship between the goods and services to be exchanged and their buyers and sellers. It is the estimate of the value of goods and services in a specific time by professional appraisers based on a specific value definition. Price refers to the amount of currency actually paid or received by the buyer and seller for the goods or services traded in a specific transaction [28]. The result of asset appraisal is a reflection of the value of the appraised object, not the actual transaction price of the appraised object.

\section{Asset Evaluation Data Analysis Based on Data Envelopment and Particle Swarm}

This article uses the IoT, PSO, and data envelopment methods to improve and develop asset evaluation plans. Since the PSO was proposed, it has received a lot of attention. Numerous research results related to PSO have 


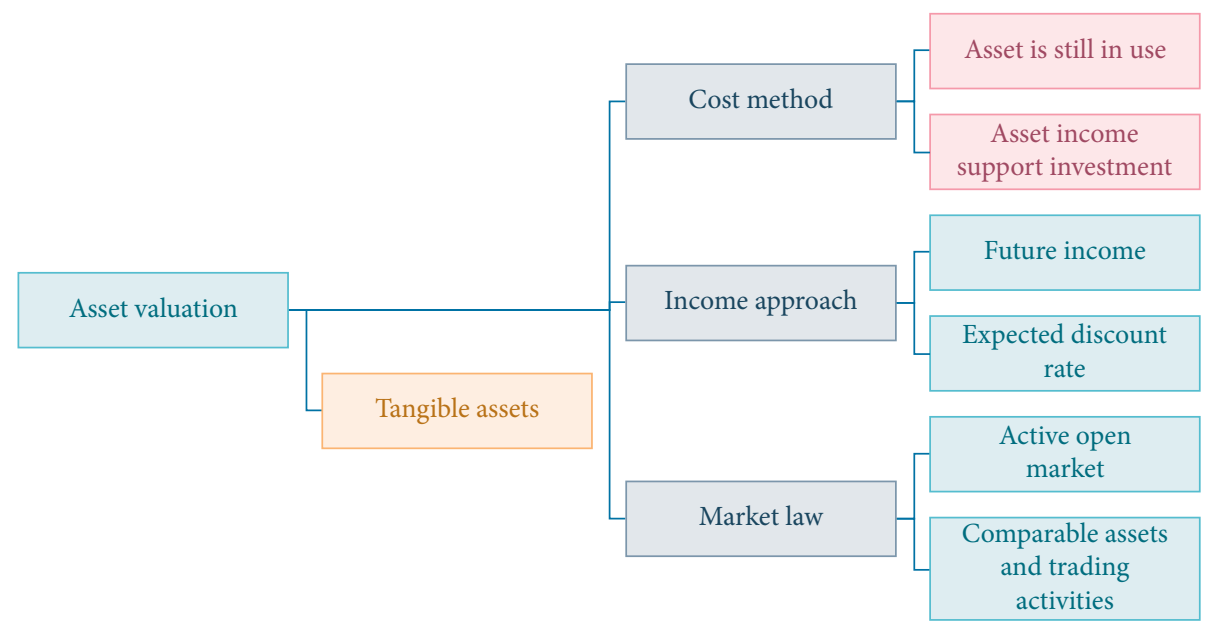

FIGURE 1: Schematic diagram of asset evaluation methods and characteristics.

appeared constantly [29, 30]. The PSO's application field has gradually expanded from the initial function optimization to a broader area, which has promoted this algorithm. PSO is mainly used in the following aspects. Function optimization is the earliest and most common application field of PSO. It applies the technical methods in financial management, accounting, engineering technology, statistics, and other disciplines according to the inherent requirements of asset evaluation to the practice of asset evaluation. They are the three major components of the asset evaluation method system. These three primary methods are actually to judge the value of assets from different time points. Based on the value at different time points, the assessed object's current value is obtained after analysis and calculation.

Based on existing research, it is found that, in some common function optimization problems, including some typical nonlinear function optimization problems, PSO has shown outstanding performance. According to bionics, PSO adopts a predator strategy. The particle of PSO is analogous to a flock of birds, and each particle is an individual among them $[31,32]$. During the foraging process, individual birds constantly adjust their foraging process based on their own experience and other birds' behavior, that is, adjusting the individual bird's speed and position in the foraging process (optimization). In this process, the individual bird's individual optimal solution can be obtained, but the bird group's optimal solution, that is, the optimal global solution, can be obtained. For the optimal solution, the following equation is used:

$$
\left\{\begin{array}{l}
v_{i j}(t+1)=v_{i j}(t)+c_{1} r_{1}(t)\left(p_{i j}(t)-x_{i j}(t)\right) \\
+c_{2} r_{2}(t)\left(p_{g j}(t)-x_{i j}(t)\right), \\
m_{i j}(t+1)=m_{i j}(t)+v_{i j}(t+1) .
\end{array}\right.
$$

PSO is used to optimize the combined algorithm to search for the minimum value more accurately [33, 34], as shown in the following equation:

$$
h_{j}=\frac{\sum_{r=1}^{s} u_{r} y_{r j 0}}{\sum_{i=1}^{m} v_{i} x_{i j 0}} .
$$

The use of PSO greatly reduces the computational complexity, makes the adjustment of the inertia weight of the wavelet basis function more rapid, and avoids being trapped locally. In this algorithm, the selection of individual position vector elements is the key to optimize the algorithm combined with the wavelet and neural network.

The wavelet analysis of the expansion parameters, translation parameters, and the combination of weights between the layers in the neural network model are used as the position vector of the particle swarm, which has good results $[35,36]$. At the same time, the particle fitness function can select the error function of the wavelet neural network, as shown in the following equation:

$$
\max h_{j 0}=\frac{\sum_{r=1}^{s} u_{r} y_{r j 0}}{\sum_{i=1}^{m} v_{i} x_{i j 0}}
$$

The algorithm will perform a random initialization at the very beginning, and the initial position of the particles often affects the quality of the result and, sometimes, directly determines the accuracy of the final result of the algorithm, as shown in the following equation:

$$
\begin{array}{ll}
\max & h_{j 0}=t \sum_{r=1}^{s} u_{r} y_{r j 0}=\sum_{r=1}^{s} \mu_{r} y_{r j 0}, \\
\text { st. } \quad\left\{\begin{array}{l}
\sum_{i=1}^{m} w_{i} x_{i j}-\sum_{r-1}^{s} \mu_{r} y r j \geq 0(j=1, \ldots, n), \\
\sum_{i=1}^{m} w_{i} x_{i j 0}=1, \\
w_{i} \geq 0(i=1, \ldots, m), \\
\mu_{r} \geq 0(r=1, \ldots, s) .
\end{array}\right.
\end{array}
$$

In PSO, the shared information contains the best information of the entire population. All particles update their next position based on their own experience and the best information of the entire population. Generally, they try their best to the current global optimal direction found in the group runs, as shown in the following equation: 
$\min \theta$,

$$
\text { st. }\left\{\begin{array}{l}
\sum_{j=1}^{n} \lambda_{j} x_{i j} \leq \theta x_{i j}(i=1, \ldots, m), \\
\sum_{j=1}^{n} \lambda_{j} y_{i j} \geq y_{r j 0}(r=1, \ldots, m), \\
\lambda_{j} \geq 0(j=1, \ldots, n) .
\end{array}\right.
$$

When solving some complex functions such as multimodality, the algorithm often falls into a local optimal solution, and it is difficult to escape this area, causing particles to stagnate in this area and appear premature, and the accuracy cannot meet the expected requirements. It can be depicted using the following equation:

$$
\text { st. }\left\{\begin{array}{l}
\frac{\sum_{r=1}^{s} u_{r} y_{r j}}{\sum_{i=1}^{m} v_{i} x_{i j}} \leq 1(j=1, \ldots, n), \\
v_{i} \geq 0(i=1, \ldots, m), \\
u_{r} \geq 0(r=1, \ldots, s) .
\end{array}\right.
$$

Using equation (3), equation (2) is transformed into an equivalent linear programming problem, as shown in the following equation:

$$
\begin{aligned}
t & =\frac{1}{\sum_{i=1}^{m} v_{i} x_{i j}}, \\
w_{i} & =t v_{i}, \\
\mu_{r} & =t u_{r} .
\end{aligned}
$$

The last step is to iteratively search for the optimized value by repeating the above procedure until the best solution is found by the particle swarm to meet the requirements, or has reached the maximum number of iterations set by the PSO.

\section{Model Simulation and Testing Process}

In this section, first we analyze the efficiency of asset valuation in Section 4.1 followed by test results of the asset evaluation system in Section 4.2.

4.1. Analysis of Asset Evaluation Efficiency. If the task is not completed, it can be analyzed from different aspects such as geographic location, task price, and member psychology. Since there are many uncertain factors in the psychological situation of members, it is difficult to carry out qualitative analysis here. Therefore, this article chooses to analyze the low task completion rate from two aspects: the spatial matching degree between the task point and the member, and the reasonableness of the pricing strategy. IoT can facilitate this scenario as it has the ability to bridge the gap between spatial matching degree and reasonableness of the pricing strategy. IoTallows the data to exchange between real objects in real time, which can be reflected in real time through legal means. Its role for asset evaluation can be realized through its perception layer. This layer is like the nerve endings of the human body's sensory organs, which can connect data in the information space with those in the physical space, i.e., asset evaluation of the communicating entities.

For asset evaluation, we make a scatter diagram of the distribution of mission points and the distribution of members. It can be seen from Figure 2 that tasks are clustered in areas with high membership density. Therefore, the spatial matching degree between the number of tasks in each area and the number of members is high, and there will be no overtasking and understaffed situations, that is, task pricing and the correlation between the spatial matching degrees is not strong. Hence, the spatial matching degree is not the main reason for the low task completion rate. The wavelet analysis of the expansion parameters, translation parameters, and the combination of weights between the layers in the neural network model are used as the position vector of the particle swarm, which has good results. At the same time, the particle fitness function can select the error function of the wavelet neural network. The scatter diagram of asset distribution and mission completion is shown in Figure 2.

The results are obtained by choosing a step size of 0.1 ; use MATLAB programming to obtain 20 sets of optimized solutions:

$$
Z_{1}=-0.002 Z_{2}-0.0869 Z_{3}+1.6 Z_{4}+0.385 Z_{5} .
$$

The completion of the 100 tasks extracted from the processing data under the new evaluation scheme is shown in Table 1.

It can be seen from Table 1 that, under the new pricing plan, the completion rate is significantly higher than that of the original plan. Therefore, it can be considered that the new pricing plan is more reasonable than the original plan, and the user acceptance is also higher. From the above, the automatic identification function and intelligent operation of the IoT achieve much higher completion rate. Broadly speaking, the IoT uses numbers and networks to represent all things, achieves not only the transmission between people but also the transmission among objects, and finally integrates the data transmitted between them.

4.2. Asset Evaluation System Test Results. The valuation of assets is an area that has received more attention in recent years. However, this work still has numerous challenges from various aspects. In order to better verify the effectiveness of the asset evaluation system established in this article, we conducted a further system performance test process on the system. The encryption time test results of the asset evaluation system are shown in Figure 3.

It can be seen from Figure 3 that when the number of plaintext bytes is less than 1 byte, the algorithm with the anonymity design has a smaller gap with the former. Please note that, in this figure, the $x$-axis represents the number of bytes and the $y$-axis represents the time in millisecond (msec). The 


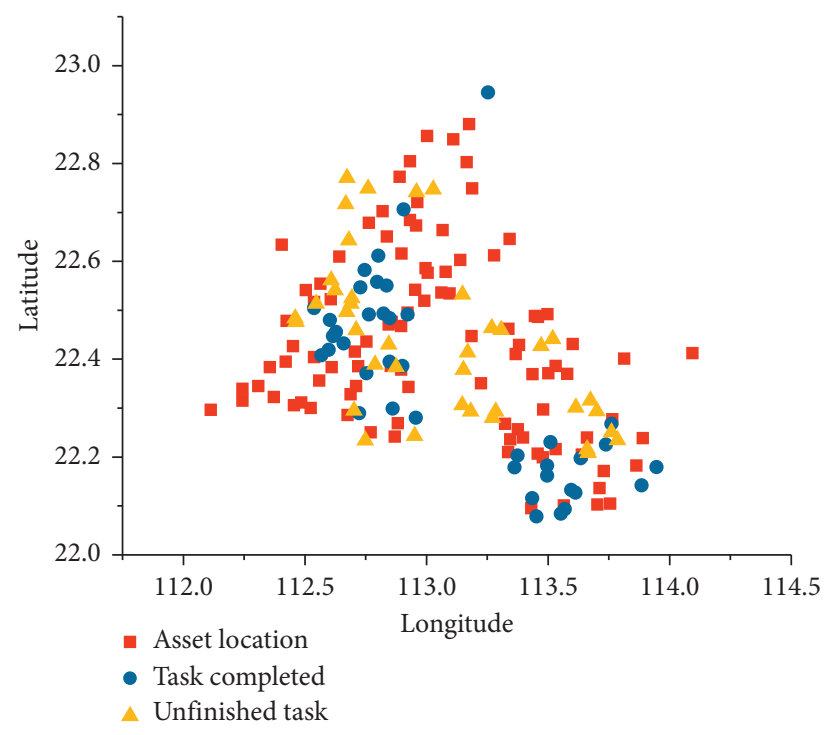

FIGURE 2: Scatter diagram of asset distribution and task completion.

TABle 1: Comparison of the completion rate of asset evaluation tasks.

\begin{tabular}{lccc}
\hline & Completed & Uncompleted & Completion rate (\%) \\
\hline New evaluation plan & 74 & 26 & 74.00 \\
Original evaluation plan & 835 & 1042 & 44.49 \\
\hline
\end{tabular}

time difference is caused by the instability of the system's encryption time when the number of plaintext bytes is small. Due to the increase in the number of cyclic groups participating in the initialization algorithm, the anonymous CIBE algorithm is at a disadvantage as a whole, but the time difference required for encryption algorithm encryption is small, so it can be said that the anonymous CIBE algorithm does not increase the computational burden on the encryption time. This algorithm can be applied to the technical methods in financial management, accounting, engineering technology, statistics, and other disciplines in accordance with the inherent requirements of asset evaluation to the practice of asset evaluation. There are three major components of the asset evaluation method system. These three basic methods are actually to judge the value of assets from different time points. Based on the value at different time points, the current value of the assessed object is obtained after analysis and calculation. Figure 4 shows the change process of the extreme value of the asset evaluation system in the evaluation process.

It can be seen from Figure 4 that the asset evaluation system has shown better results and performance in the extreme value test. This shows that the system can effectively carry system fluctuations under different conditions. The wavelet analysis of the expansion parameters, translation parameters, and the combination of weights between the layers in the neural network model are used as the position vector of the particle swarm, which has good results. At the same time, the particle fitness function can select the error function of the wavelet neural network.

For the asset evaluation system established in this article, we test the score distribution of the system for different assets. Therefore, this article chooses to analyze the low task completion rate from two aspects: the spatial matching degree between the task point and the member, and the reasonableness of the pricing strategy. The distribution of the scores of different assets at the time of evaluation is shown in Figure 5.

It can be seen from Figure 5 that the asset evaluation system has shown better results and performance for the score distribution of different assets during evaluation. The comparison is first made based on longitudinal comparison, which uses multiple sets of data to test how the optimized algorithm compares with the preoptimized algorithm and whether the similar optimized algorithms have changed, and whether the performance is more suitable for the edge environment of the IoT. 


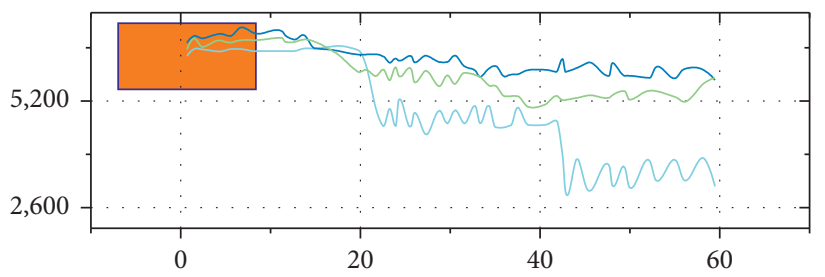

(a)

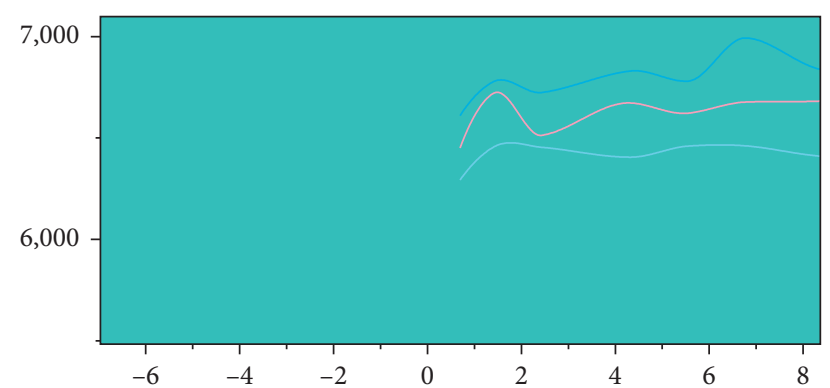

(b)

FIgURE 3: The encryption time test chart of the asset evaluation system.

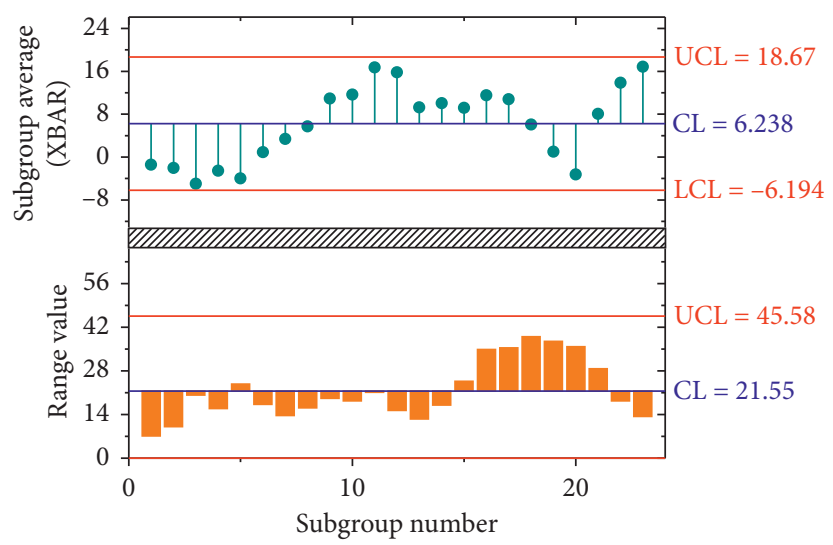

FIGURE 4: Changes in the range of the asset appraisal system during the appraisal process.

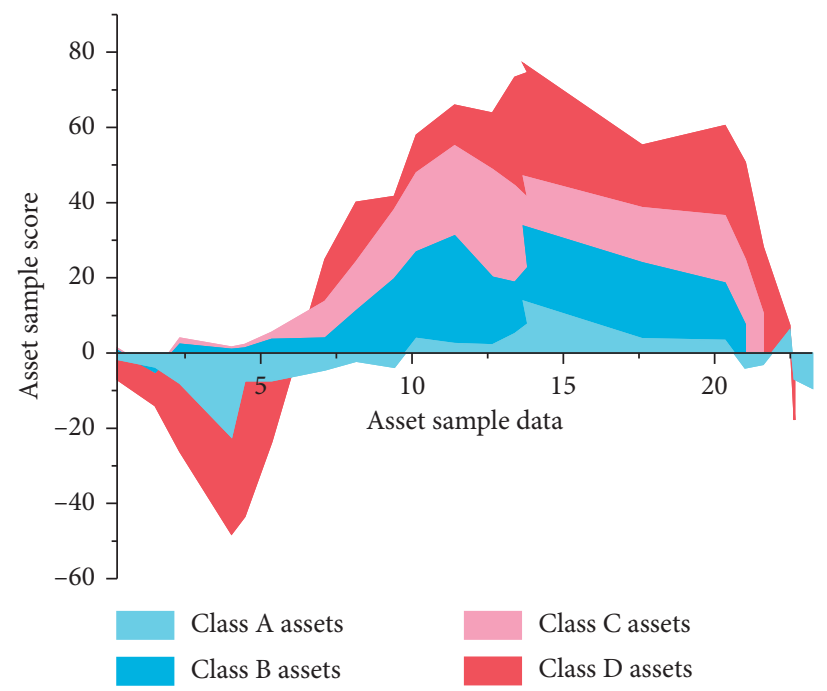

FIGURE 5: The distribution of scores of different assets at the time of evaluation.

\section{Conclusions}

With the advent of globalization and the era of knowledge economy, the profoundly changing domestic and international environment, on the one hand, provides extensive development space for the asset appraisal industry but also brings a lot of new challenges to the healthy development of the asset appraisal industry on the other hand. Business evaluation has begun to transform from traditional businesses to emerging businesses due to the technological 
trends and the emergence of the Internet of Things (IoT). However, there are numerous challenges for effective asset evaluation from a technology perspective. In this article, an asset evaluation framework was designed to use the data envelopment methods, IoT, and particle swarm optimization (PSO) algorithm. This framework can provide an allweather and no-delay preparation for asset appraisal. Data envelopment and PSO algorithm ensure the cleaning and reclassification of asset data. We verify the effectiveness and reasonable practicability of the model by comparing experimental simulation and manual evaluation. Although this article has carried out a more in-depth study of asset evaluation, it still has numerous gaps to be filled due to the limitation of personal knowledge level. In the future, we will further research asset appraisal and provide scientific advice for the asset appraisal industry's healthy development.

\section{Data Availability}

The data used to support the findings of the study are available from the corresponding author upon request.

\section{Conflicts of Interest}

The authors declare that they have no conflicts of interest.

\section{References}

[1] B. J. Wu, A. X. He, A. Y. Le et al., "How earthquake-induced direct economic losses change with earthquake magnitude, asset value, residential building structural type and physical environment: an elasticity perspective," Journal of Environmental Management, vol. 231, pp. 321-328, 2019.

[2] H. Masset, M. Z. Esteki, E. Dimitriadou et al., "Multi-centre evaluation of a comprehensive preimplantation genetic test through haplotyping-by-sequencing," Human Reproduction, vol. 34, no. 8, 2019.

[3] P. Lin and X.-X. Yuan, "A two-time-scale point process model of water main breaks for infrastructure asset management," Water Research, vol. 150, no. 1, pp. 296-309, 2019.

[4] F. Chasset and C. Francès, "Current concepts and future approaches in the treatment of autaneous lupus trythematosus: a comprehensive review," Drugs, vol. 79, no. 11, 2019.

[5] D. P. Bigelow and T. Kuethe, "A tale of two corders: use value assessment, land development, and irrigation envestment," American Journal of Agricultural Economics, vol. 102, no. 4, pp. 1404-1424, 2020.

[6] J. Abreu-Gomez, D. Walker, T. Alotaibi, M. D. F. McInnes, T. A. Flood, and N. Schieda, "Effect of observation size and apparent diffusion coefficient (ADC) value in PI-RADS v2.1 assessment category 4 and 5 observations compared to adverse pathological outcomes," European Radiology, vol. 30, no. 8, pp. 4251-4261, 2020.

[7] F. Lais, G. Navarro, S. M. Leite et al., "Pathways linking environmental risks, polygenic risk score to schizophrenia and psychotic experiences," A Brazilian Cohort of Children and Adolescents Schizophrenia Bulletin, vol. 120, no. 1, p. S24, 2020.

[8] K. Kaddoura, T. Zayed, Z. Chen et al., "Optimized maintenance model for wastewater treatment plants," Canadian Journal of Civil Engineering, vol. 30, pp. 1-47, 2020.
[9] S. Sannigrahi, S. Chakraborti, P. K. Joshi et al., "Ecosystem service value assessment of a natural reserve region for strengthening protection and conservation," Journal of Environmental Management, vol. 244, no. 15, pp. 208-227, 2019.

[10] A. Aslam, U. Mehmood, M. H. Arshad et al., "Dye-sensitized solar cells (DSSCs) as a potential photovoltaic technology for the self-powered internet of things (IoTs) applications," Solar Energy, vol. 207, pp. 874-892, 2020.

[11] B. M. Elhalawany, R. Ruby, and K. Wu, "D2D communication for enabling internet-of-things: outage probability analysis," IEEE Transactions on Vehicular Technology, vol. 1, no. 99, 2019.

[12] S. Gupta, E. M. Johnson, J. G. Peacock et al., "Radiology, mobile devices, and internet of things (IoT)," Journal of Digital Imaging, vol. 33, no. 12, 2020.

[13] Y. Qian, L. Ma, and X. Liang, "The performance of chirp signal used in LEO satellite internet of things," IEEE Communications Letters, vol. 23, no. 8, 2019.

[14] B. Pourghebleh, V. Hayyolalam, and A. A. Anvigh, "Service discovery in the Internet of Things: review of current trends and research challenges," Wireless Networks, vol. 26, no. 7, 2020.

[15] A. C. Faleye, A. A. Adegoke, K. Ramluckan et al., "Everything you wanted to know about smart cities: the internet of things is the backbone," Science of the Total Environment, vol. 5, no. 3, pp. 60-70, 2019.

[16] C. Michie, I. Andonovic, C. Davison et al., "The internet of things enhancing animal welfare and farm operational efficiency," Journal of Dairy Research, vol. 87, no. S1, pp. 1-8, 2020.

[17] U. Tariq, A. Ibrahim, T. Ahmad, Y. Bouteraa, and A. Elmogy, "Blockchain in internet-of-things: a necessity framework for security, reliability, transparency, immutability and liability," IET Communications, vol. 13, no. 19, pp. 3187-3192, 2019.

[18] M. Usman, A. Jolfaei, and M. A. Jan, "RaSEC: an intelligent framework for reliable and secure multilevel edge computing in industrial environments," IEEE Transactions on Industry Applications, vol. 56, no. 4, pp. 4543-4551, 2020.

[19] Y. Yang, W. J. Xue, and J. Liu, "Efficiency evaluation of county-level public hospitals in Hainan, China: a four-stage data envelope analysis model based on panel data," Chinese Medical Journal, vol. 134, pp. 876-878, 2021.

[20] H. Khan, M. A. Jan, M. Alam, and W. Dghais, "A channel borrowing approach for cluster-based hierarchical wireless sensor networks," Mobile Networks and Applications, vol. 24, no. 4, pp. 1306-1316, 2019.

[21] Y. J. Chen and S. E. Chang, "CMOS cook modulator with fast envelope transient," Electronics Letters, vol. 55, no. 17, 2019.

[22] G. N. Eastep, R. H. Ghanam, T. J. Green, and J. S. Saad, "Structural characterization of HIV-1 matrix mutants implicated in envelope incorporation," Journal of Biological Chemistry, vol. 296, no. 2, Article ID 100321, 2021.

[23] W. Yao, F. Khan, M. A. Jan et al., "Artificial intelligence-based load optimization in cognitive internet of things," Neural Computing and Applications, vol. 32, pp. 1-11, 2020.

[24] X. Li and Y. Liu, "A quick algorithm for evaluation of minimum zone circles from polar coordinate data," Review of Scientific Instruments, vol. 90, no. 12, Article ID 125114, 2019.

[25] M. Ibrar, A. Akbar, R. Jan et al., "ARTNet: AI-based resource allocation and task affloading in a reconfigurable internet of vehicular networks," IEEE Transactions on Network Science and Engineering, vol. 44, p. 1, 2020.

[26] S. Pollmann, A. Springer, S. Rustgi et al., "Substrate channeling in oxylipin biosynthesis through a protein complex in 
the plastid envelope of Arabidopsis thaliana," Journal of Experimental Botany, vol. 70, no. 19, pp. 1483-1495, 2019.

[27] H. W. Yen, S. Takakuwa, P. G. Gu et al., "Signs of outflow feedback from a nearby young stellar object on the protostellar envelope around HL tau," 2019.

[28] L. Doan, S. Ramstedt, W. Vlemmings et al., "The extended molecular envelope of the asymptotic giant branch star $\$ \backslash$ î $\{1\} \$$ gruis as seen by ALMA II. the spiral-outflow observed at high-angular resolution," Astronomy and Astrophysics, vol. 20, pp. 1-10, 2019.

[29] B. Miszalski and V. Manick, "The post-common-envelope binary nucleus of the planetary nebula IC 4776: neither an anomalously long orbital period nor a wolf-rayet binary," Monthly Notices of the Royal Astronomical Society, vol. 14, no. 1, p. 1, 2019.

[30] M. Bertagnolli and J. Tabernero, "Value assessment frameworks in oncology: championing concordance through shared standards," Annals of Oncology, vol. 30, pp. 505-506, 2019.

[31] C. V. Gonzalez, I. Herraiz, J. Delgado et al., "OP10.05: oetal growth assessment and perinatal outcomes associated with extremely high sFlt/PlGF value," Ultrasound in Obstetrics and Gynecology, vol. 54, no. S1, pp. 116-117, 2019.

[32] A. Pichon-Riviere, S. Garcia-Marti, W. Oortwijn, F. Augustovski, and L. Sampietro-Colom, "Defining the value of health technologies in latin America: cevelopments in value frameworks to inform the cllocation of healthcare sesources," International Journal of Technology Assessment in Health Care, vol. 35, no. 1, pp. 64-68, 2019.

[33] P. Bahri, D. R. Morales, A. Inoubli et al., "Proposals for engaging fatients and healthcare professionals in risk minimisation from an analysis of dtakeholder input to the EU ialproate assessment using the novel analysing stakeholder hafety engagement tool (ASSET)," Drug Safety, vol. 10, pp. 1-44, 2021.

[34] D. Schlicke, N. V. Tue, J. Gomes, C. Sousa, and M. Azenha, "Analytical assessment of restraint forces and crack widths in end-restrained building slabs," Engineering Structures, vol. 224, Article ID 111218, 2020.

[35] M. N. Scheu, L. Tremps, U. Smolka, A. Kolios, and F. Brennan, "A systematic failure mode effects and criticality analysis for offshore wind turbine systems towards integrated condition based maintenance strategies," Ocean Engineering, vol. 176, no. 15, pp. 118-133, 2019.

[36] V. Kunniyoor, P. Singh, and K. Nadella, "Value of closed-cycle gas turbines with design assessment," Applied Energy, vol. 269, Article ID 114950, 2020. 